\title{
ACTIVITIES ON TERMINAL ANTENNAS IN ACE2
}

\author{
Marta Martínez-Vázquez ${ }^{(1)}$, Raquel Serrano ${ }^{(2)}$, Jan Carlsson ${ }^{(3)}$, Anja K. Skrivervik ${ }^{(4)}$ \\ ${ }^{(1)}$ IMST GmbH, Carl-Friedrich-Gauß-Str. 2, D-47475 Kamp-Lintfort, Germany, martinez@imst.de \\ ${ }^{(2)}$ Universitat Politecnica de Catalunya, Jordi Gerona 1-3, 08034 Barcelona, Spain, raquel.serrano@tsc.upc.edu \\ ${ }^{(3)}$ Chalmers Tekniska Högskola AB,Horsalvägen 11, S-41296 Gothenburg, jan.carlsson@sp.se \\ ${ }^{(4)}$ LEMA, Ecole Polytechnique Fédérale de Lausanne, Station 11, CH-1015 Lausanne, anja.skrivervik@epfl.ch
}

\begin{abstract}
This paper presents the work carried out in the ACE Network of Excellence regarding small antennas. Three aspects are covered in three projects : Small antenna technologies, small terminal antenna technologies and benchmarking of small terminal antennas measurement facilities. The aim is to identify the newest trends in antenna design and measurement for personal communications devices, and suggest novel solutions and design methodologies for various applications.
\end{abstract}

\section{INTRODUCTION}

The Networks of Excellence were introduced in the $6^{\text {th }}$ Framework Program of the European Union as a new instrument to integrate and structure research activities around Europe, to increase the relevance of their results by avoiding overlapping [1]. Among them, the Antenna Centre of Excellence (ACE, 2004-2005) was created, complementing the activities of the COST 284 action: "Innovative Antennas for Emerging Terrestrial \& Space-based Applications" [2]. ACE 2 (2006-2007) follows up the work started in ACE, to try to achieve a durable structuring. In this contribution we give an overview on the activities on small and terminal antennas done within ACE and ACE 2. The work has been divided into three main domains : Small antenna technologies, terminal antenna technologies and the benchmarking of small terminal antennas measurement facilities. The two first aspects cover the fundamental physics behind electrically small antennas, design, simulation and the prediction of new trends, whereas the third is related to the specific problematic of small antenna measurements.

\section{SMALL ANTENNA TECHNOLOGIES}

The evolution of the wireless communication services in the past decades have put new and interesting challenges to antenna designers : not only does the physical space allotted to antennas decrease with each new generation of handsets, but more and more services (working in different frequency bands) have to share the same space. This evolution, along with the apparition of services like RF-IDs and sensing require the use of devices with a high energy efficiency. The future, therefore, lies in small, internally mounted, antennas that are able to work well across a large bandwidth. Neither the radiation mechanism nor the design of small antennas in modern applications such as Ultra Wide Band, MIMO systems and sensor networks, can be understood with traditional radiation theories for small antennas. New antenna requirements entail new modeling tools and new design technologies. Since the compactness of the RF transmitter and receiver is increasing, the effects of integrating the antennas with the active element are studied. The newest trends in compact small antenna design have to be rapidly detected. The aim of our work is to optimize the existing design methodologies, and make use of the possibilities offered by new methodologies applied to small antennas.

Different downsizing techniques and their implication on antenna performances have been studied in literature see for instance [3, 4], and can be summarized as follows :

- The use of parasitic resonators to obtain a double resonant behavior

- Slit etching as a way of increasing the electrical path while maintaining physical size.

- Short-circuiting walls to utilize the half-size reduction factor.

- Dielectric loading, especially in packaged applications, for further size reduction at the cost of lower efficiency.

- Loading with lumped reactive circuit elements like capacitors or inductors, at the cost of a narrower bandwidth

These downsizing solutions are however not sufficient when the new applications (UWB, MIMO, RF-ID) are considered, as the quality of these devices require 
additional criteria : UWB systems for instance asks from antennas a very large frequency band, but also a very short impulse response, a phase centre which remains fixed over the entire frequency band and a high radiation efficiency. An important indicator of the quality of a MIMO system is the channel capacity. This capacity depends on many factors, namely the number of transmit antennas and their spacing, the number of receive antennas and their spacing and the multipath environment and, in particular the spread of direction of arrival angles. As result the antenna is getting more and more interrelated with the system parameters (RF active parameters and channel parameters) which leads to the recommendation of considering the antenna as a central part of the system, designing its parameters in the context of the full system optimization. In consequence, the actual modeling techniques basically formulated for a single port resonant antenna are not adequate anymore, and a deep approach to the requirements, modeling and design techniques for the future small antennas must be carried out, which implies an special effort. An intense ongoing research work is being done in this direction.

As an illustration to the problems sated above, let us consider the design, measurement and modeling problems induced by a new family of antennas, wearable antennas. Two main types of such antennas exists, flexible one, which are made of printed antennas on textiles, or rigid, button shape antennas. In both cases, new challenges are posed to the antenna designer

- The dielectric substrates used are new (mostly textiles), and specific dedicated measurement procedures have to be set up to obtain their dielectric constant and loss factor

- The entire system will be worn by the user, but should also be functional without the presence of the user, which means the design has to be robust to a drastic change of environment

- The system has to be characterized in presence and absence of the user

The same kind of consideration can be done for a large number of upcoming products and services.

\section{SMALL TERMINAL ANTENNA TECHNOLOGIES}

This activity focuses on two main directions : the identification of emerging needs, trends and standards for the future on one hand, and the study of upcoming novel application on the other.

\subsection{Future trends}

This first focus can be subdivided in five actions :

- The follow-up of the review of novel applications, technologies and materials.

- The assessment of the effect of deep integration of the antenna into the system, with a special focus on the coupling mechanism between the radiators themselves and the surrounding devices

- The review and assessment of design methodologies

- The review of dedicated analysis tools

- The review of dedicated optimization tools

Once these reviews and critical assessments are finalized, the result obtained will be summarized into a new set of guidelines.

\subsection{Small terminal applications}

This second area of focus is centered on the problems created new small terminal applications.

\section{Multi-antenna systems and MIMO}

the optimization of multi-antenna topologies should be done taking into account the MIMO system performance point of view. Indeed, in new systems such as UMTS, multi-element antenna structures in the terminal are a popular way to achieve antenna diversity, in order to improve the link reliability needed for reaching high data rates.

It has been shown that MIMO wireless systems can provide increased capacity in rich multipath environments [5]. MIMO antenna systems can be used in a mobile terminal to fight the impairments of the propagation channel, and thus obtain additional gain over other well-established classical strategies.

This is also a very interesting solution for nomadic applications, such as laptops in a WiMAX environment [6]. In a small mobile terminal the number of antenna elements may range typically from two in a small handset, to four in a PDA, and up to sixteen or even more, in a laptop computer [7]. A realistic number of antenna elements placed on the quite small mobile terminals is probably less than five. In this context especially dual-polarized antenna elements are interesting, as they enable locating two antennas in the same volume. Moreover, as $2 \mathrm{G}$ and $3 \mathrm{G}$ mobile networks will continue to coexist during a certain period, one antenna for each communication standards will have to be integrated above the same terminal [8] or at least, when the radio front-end modules will reach 
maturity, a multi-band antenna covering the involved standards [9].

Digital broadcasting, UWB systems

One of the latest upcoming trends in mobile communications technology is the reception of digitalTV on mobile terminals. Besides the reception of public video streams it would also be possible to use this broadcast technology for other large-bandwidth data traffic (in the reception side), such as general or commercial localized information distribution. Antennas for DAB terminals must therefore be adapted to cover these bands with a reasonable performance.

\section{RFIDs}

The great appeal of RFID technology is that it allows information to be stored and read without requiring either physical contact or a line of sight between the tag and the reader by using electromagnetic waves. The key design challenge is achieving tag antennas with sufficiently high gain. The tags are furthermore required to be small in size and mechanically robust against vibrations. In spite of the downsizing techniques that are used in order to design such meandered antennas, their dimensions are still quite large in comparison with the rest of the circuitry.

\section{Body Area networks}

Future mobile communications systems will include so-called body-centric configurations, with modules distributed all over the body of the human user, forming a "Body Area Network" (BAN). Such wireless networks should provide a communication system available in all circumstances, with a high degree of reconfigurability, yet unobtrusive to the wearer. Such on-body networks have to respond to unique problems, related to the rapid changes in the communications channel. Indeed, the later will change depending on the subject carrying the equipment, and even on the position of the wearer

\section{In Body applications}

Designing antennas for in-body applications is very challenging, due to reduced antenna efficiency, the need to reduce the antenna size, the impact of the environment and the multipath losses. However, the apparition in medical applications of ultra-small devices (which can be injected into a vein, for instance) and the need to be able to communicate with them is leading to need of ultra miniaturized antennas optimized for working in lossy media. Indeed, the frequency bands foreseen for these applications are the standard MICS band (402-405 MHZ), the Body Area Network (BAN) band (433-434 MHz) and the ISM band at $2.45 \mathrm{GHz}$, which means that the allowable antenna size for an implantable device can be as small as $0.03 \lambda$.

There are in literature some works about implantable antennas. Most of them concern antennas for therapeutic applications [10-12] (hyperthermia, balloon angioplasty, ...) or for sensing applications. In both cases, the antennas works in its near field, and propagation over a certain distance is not an issue. The situation is completely different in telemetry applications, where the antenna is used to transmit diagnostic information $[13,14]$ : in this case, features like the radiation efficiency and the bandwidth become essential in order to provide transmission over a large enough range with a high enough data rate.

\section{BENCHMARKING OF SMALL TERMINAL ANTENNAS MEASUREMENT FACILITIES}

Benchmarking of test facilities is a work package included in the small terminals and smart antennas activity within ACE - Antenna Centre of Excellence [1]. Among the main objectives of the work package are the definition of test cases for the characterization of terminal antennas and the comparison of results from measurements performed at different antenna test facilities around Europe.

Usually, antennas are characterized by their radiation patterns. This is useful when the lobes are narrow and there is line of sight between the two sides. For the case of terminal antennas, e.g. mobile phone and PDA antennas, the environment varies with time and is complex with several scatterers, so it can only be described statistically. A better way to describe the radiation characteristics of terminal antennas is to use the total radiation efficiency. This includes contributions from losses in the antenna itself, losses in the near-in environment of the antenna and impedance mismatch. When the impedance mismatch is not included, we call the remaining factor the radiation efficiency. The total radiation efficiency is a correct measure of the quality of antennas that are designed for use in a so called uniform multi-path environment, where there is no "line of sight" to the base station and reflected and scattered waves come in with equal probability from all directions in space. Total radiation efficiency can be measured using many different methods, e.g. by measuring the $3 \mathrm{D}$ radiation pattern and integrating, using a reverberation chamber [15] or a Wheeler cap [16]. Therefore, a test case that should be used for benchmarking of different test facilities must be constructed so that it can be used in different set-ups and at the same time be configurable so that several test cases can be defined. It is also an advantage if the test case is simple enough to allow numerical simulations 
with good accuracy for enabling additional comparisons. The developed test fixture fulfils these requirements and can be used for both efficiency and diversity gain measurements.

For the active devices total radiated power (TRP) and total isotropic receiving sensitivity (TIS) are measured. TRP is measured for two test cases: a GSM phone with special software allowing it to radiate at a constant power level, and a specially developed $1.875 \mathrm{GHz}$ device. Sensitivity is measured for a standard GSM phone, and therefore a base station emulator is needed for these measurements. Both the TRP and the TIS measurements for the GSM phones are done with the phone both in a free space position and in a talk position close to a head phantom. TRP for the 1.785 $\mathrm{GHz}$ device is only measured for free space conditions. An overview of the different test cases can be seen in figure 1 .

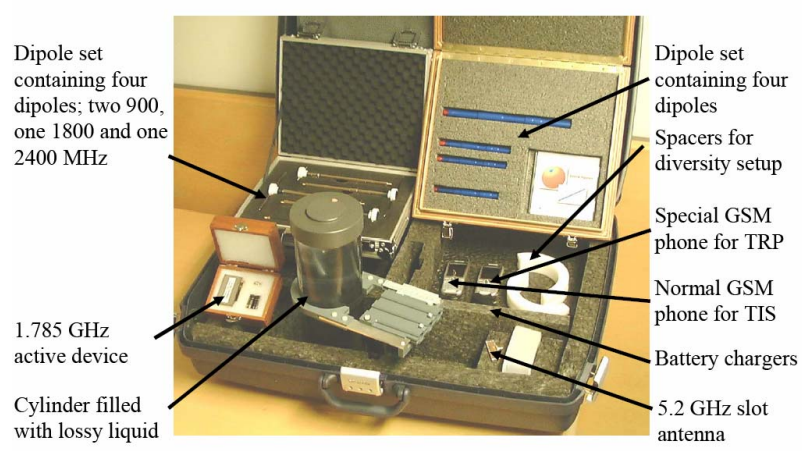

Figure 1 : Benchmarking test antennas

As an example of results that came out of the comparison of the different testing equipment, the total radiation efficiency for a $5.2 \mathrm{GHz}$ slot antenna measured at ten facilities is shown in Fig.2. The maximum deviation from mean is of $2.7 \mathrm{~dB}$.

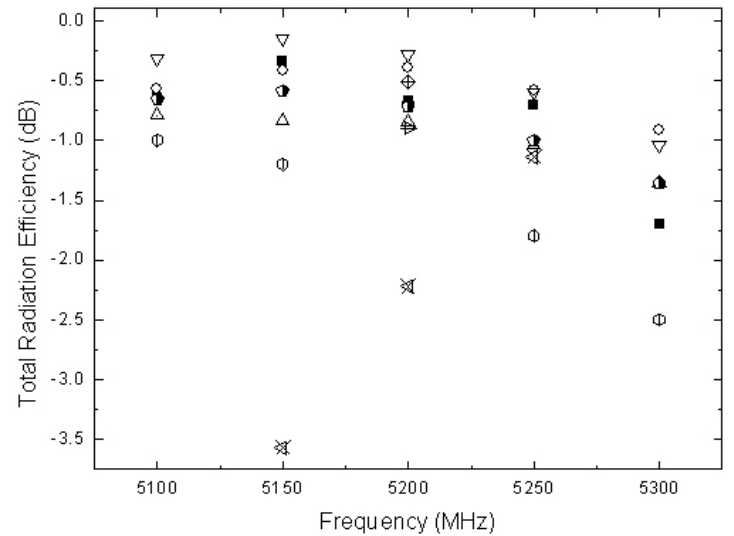

Figure 2 :Benchmark antenna and benchmarking results

\section{REFERENCES}

[1] www.ist-ace.org.

[2] www.cost284.com

[3] A.K. Skrivervik, J-F. Zürcher, O. Staub, J.R. Mosig, "PCS antenna design: the challenge of miniaturization", IEEE Antennas and Propagation Magazine, vol. 43, Issue 4, August 2001, pp. 12-27.

[4] Jofre, L., Cetiner, B.A. and De Flaviis, F. "Miniature Multi-Element Antenna for Wireless Communications", IEEE Trans. Antennas Propagat. Vol. 50. pp 658-669, May, 2002.

[5] Foschini, G.J. and Gans, M.J., "On Limits of Wireless Communications in a Fading Environment when Using Multiple Antennas", Wireless Personal Communications, Vol. 6, No. 3, March 1998, pp. 311335.

[6] Mobile WiMAX - Part I: A Technical Overview and Performance Evaluation, WiMAX Forum, http://www.wimaxforum.org

[7] Antoniuk, J., Moreira, A. and Peixeiro, C., "MultiElement Patch Antenna Integration into Laptops for Multi-Standard Applications", IEEE Antennas and Propagation Society Symposium, Washington DC, July 2005.

[8] Diallo, A., Luxey, C., Le Thuc, P., Staraj, R. and Kossiavas, G., 'Reduction of the mutual coupling between two planar inverted-F antennas working in close radiocommunication standards', 18th International Conference on Applied Electromagnetics and Communications (IceCom), Dubrovnik, Croatia, October 2005

[9] Ciais, P., Staraj, R., Kossiavas, G. and Luxey, C., 'Design of an Internal Quad-Band Antenna for Mobile Phones', IEEE Microwave and Wireless Components Letters, Vol. 14, no. 4, April 2004, pp. 148-150.

[10] A. Rosen, "Microwave application in cancer therapy, cardiology and measurement techniques: A short overview", IEEE MTT Newslett., pp17-20, Fall 1990.

[12] A. Guy, J.F. Lehman and J.B. Stonebridge, "Therapeutic applications of electromangetic power", Proc. IEEE, vol. 62, pp- 55-75, Jan. 1974

[13] D.M. Furse and M.F. Iskander, "Three-dimensonal electromangetic power deposition in tumors using interstitial antenna arrays", IEEE Trans. Biomed. Eng.,

$\rightarrow$ Lab 2 vol. 36, pp. 977-986, Oct 1989.

$\stackrel{\star}{\star}$ Lab 3 La14] B.M. Steinhaus, R.E: Smith and P. Crosby, "the role of - Lab5 telecommunications in future implantable device " Lab 6 telecommunications in future implantable device

Lab 8

$\rightarrow$ Lab 9 1994, pp. 1013-1014,

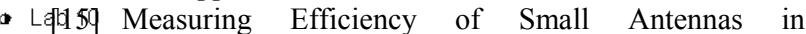
Reverberation Chambers," IEEE AP-S International Symposium and URSI Science Meeting, Columbus, Ohio, USA, June $22-27,2003$.

[16] H.A. Wheeler, "The Radiansphere Around a Small Antenna," Proceedings of the IRE, Vol. 47, August 1959. 